\title{
Living alone and mortality among older people in Västerbotten County in Sweden: a survey and register-based longitudinal study
}

Nawi $\mathrm{Ng}^{1,2,3^{*}}$ (D) Ailiana Santosa ${ }^{2,3}$, Lars Weinehall ${ }^{1}$ and Gunnar Malmberg ${ }^{2,4}$

\begin{abstract}
Background: Living alone is increasingly common and has been depicted as an important cause of mortality. We examined the association between living alone and mortality risks among older men and women in northern Sweden, by linking two unique longitudinal datasets.

Methods: We used the Linnaeus database, which links several population registers on socioeconomic and health. This register-based study included 22,226 men and 23,390 women aged 50 and 60 years in Västerbotten County who had participated in the Västerbotten Intervention Program (VIP) during 1990-2006, with a total of 445,823 person-years of observation. We conducted Cox-proportional hazard regression to assess the risk of living alone on the mortality that was observed between 1990 and 2015, controlling for socio-demographic factors, chronic disease risk factors and access to social capital.

Results: Older men and women who lived alone with no children at home were at a significantly higher risk of death compared to married/cohabiting couples with children at home (with an adjusted hazard ratio of 1.38, 95\% $\mathrm{Cl}$ of 1.26-1.50 in men and 1.27, 95\% Cl of 1.13-1.42 in women). Living alone was an even stronger factor than the well-established chronic disease risk factors and a lack of access to social capital.

Conclusions: A significant association between living alone and mortality among the older adult population in Sweden was observed. Providing good social support for older people is important in preventing the negative health impact of living alone.
\end{abstract}

Keywords: Living alone, Older people, Social support, Family network, Living arrangement, Deaths

\section{Introduction}

Living alone is becoming increasingly common in highincome countries, due to for instance trends of longevity, high divorce rates, low rates of intergenerational coresidence, and high rates of widowhood [1-3]. The trends are more advanced in some countries, where Sweden reports the highest prevalence of living alone in Europe with more than $50 \%$ of the households being single household [4]. At the same time, it has been claimed that living alone is a

\footnotetext{
* Correspondence: nawi.ng@umu.se

${ }^{1}$ Department of Epidemiology and Global Health, Faculty of Medicine, Umeå University, Umeå, Sweden

${ }^{2}$ Centre for Demographic and Ageing Research, Umeå University, Umeå,

Sweden

Full list of author information is available at the end of the article
}

major risk factor for mortality and morbidity not least for the older people for several reasons [5-7]. It may cause social isolation, and if not compensated by other forms of social capital can result in feeling of loneliness and depression with negative consequences on health and survival $[8,9]$. The effect of social isolation on mortality is comparable to traditional clinical risk factors $[7,10]$. People in single households maybe more prone to adopt poor health behaviours $[9,11]$. Living alone is also more costly and may add to the economic burdens of people with low socio-economic position. In addition, unemployment and retirement may lead to social isolation with more serious consequences on health and mortality for those living alone [12]. Moreover, people living alone are more likely to experience unmet care needs [13]. 
In this study, we examine the association between living alone and mortality among men and women aged 50 and 60 years in Västerbotten County. This study provides a novel contribution to the literature by linking data from the comprehensive data of the Västerbotten Intervention Programme (VIP) with Swedish national register data that allow a longitudinal follow-up of survival among the Swedish older population. By including various covariates in the analysis, we have explored the association between living alone and mortality after adjusting for lifestyle factors, socioeconomic position, ill-health and access to social capital. Being a forerunner in the extent of living alone, the Swedish case is special but interesting since it may inform us about the coming trends in other European countries and elsewhere.

\section{Methods}

\section{Study design and population}

We used the longitudinal information from the Linnaeus database, which links the Swedish population register, socio-economic data, hospitalisation and the Swedish death register at national level with the comprehensive VIP data (see below) during the years 1990-2006. Details about the Linnaeus database have been presented elsewhere [14].

In this study, we analysed the population in Västerbotten County who participated in the VIP. In brief, the VIP is a combined individual- and population-based health intervention programme for reducing the burden of cardiovascular disease risk factors. Individuals who turn 40, 50, and 60 years old in the county were invited for health screening which included questionnaire, anthropometric and blood pressure measurement, and blood sampling. Trained nurses provided feedback to each individual in a counselling session [15]. In this paper, we focused on individuals aged 50 and 60 years who had participated for the first time in the VIP during the years 1990-2006 ( $n=45,616$, with a total follow-up time of 445,823 person-years). As the VIP data is linked to the population register data in the Linnaeus database, we obtained their socioeconomic and living arrangement data in the same year as when the individuals participated in the VIP examination, as well as death date for those who died.

\section{Outcome variables}

We recorded deaths occurring among the study participants until October 2015.

\section{Independent variables}

The main independent variable of interest was living alone, constructed from family type and living arrangement data. We categorised the 'living alone' variable into four categories of (i) married or cohabited with children living at home, (ii) married or cohabited without children living at home, (iii) single parent with children living at home, and (iv) single parent without children living at home. We defined the participants as 'living alone' if they belonged to the last category.

\section{Sociodemographic variables}

Socio-demographic variables included were: sex (men and women); age group (50,60 years); and education level (basic, middle, and high education). The employment variable was categorised into full-time employment, intermittent employment, mostly unemployed, fully unemployed, partly retired, and fully retired.

\section{Chronic disease risk factor variables}

Smoking status and smokeless tobacco (referred to as snus in Sweden) users were assessed based on the question "Do you currently smoke?" and "Do you currently use snus?". We dichotomised the responses into 'never/non-current' and 'current smokers/snus users'. Individuals who responded positively to at least two of the CAGE questions were categorised as having a risk of alcohol dependency. CAGE is the acronym of the four questions in the CAGE questionnaire, which measures if the respondents: had felt the need to Cut down on drinking, had been Annoyed by people who criticised the drinking, had ever felt Guilt about drinking, and had felt the need to drink first thing in the morning as Eyeopener [16]. Physical activity was measured using questions on commuting, recreational time, and physical exercise. Responses from these physical activity questions were analysed and later classified into 'sedentary', 'moderate' or 'physically active'. Details about the construction of the physical activity variable have been published elsewhere [17]. Body mass index (BMI) was categorised into 'under/normal weight' $\left(\mathrm{BMI}<25 \mathrm{~kg} / \mathrm{m}^{2}\right)$ and 'overweight or obese' (BMI $\geq 25 \mathrm{~kg} /$ $\mathrm{m}^{2}$ ). Respondents were categorised as diabetic if they had fasting blood glucose of $\geq 7 \mathrm{mmol} / \mathrm{L}$ or 2 -h blood glucose of $\geq 12.2 \mathrm{mmol} / \mathrm{L}$. Cholesterol level was dichotomised into 'normal' or 'hypercholesterolemia' (if blood cholesterol $\geq 6.5$ $\mathrm{mmol} / \mathrm{L}$ ). Respondents were classified as having high blood pressure if the average systolic blood pressure was $\geq 140$ $\mathrm{mmHg}$ or the diastolic blood pressure was $\geq 90 \mathrm{mmHg}$, or if they had been taking blood pressure-lowering medication in the last 14 days.

\section{Social capital variables}

Social capital was measured based on self-reported information on social participation and informal socialising. The respondents were asked if they participated in any leisure-time activity or volunteer organisation during the last year, and if so, how often. They were also asked the number of people: (i) who they know and interact with; (ii) who can come to their house anytime and feel at home; and (iii) who were family or friends with whom they can speak their mind openly. Based on their responses, participants were categorised into having 'low', 'medium' and 'high' levels of social 
participation and informal socialising. The details on how social capital was constructed has been published earlier [18].

\section{Statistical analyses}

As many of the chronic disease risk factors are related to each other, the inclusion of the factors as separate variables in a multivariable regression analysis will cause the problem of multicollinearity. We, therefore, performed a multivariate analysis to reduce the dimension of the risk factor data. We created an index of chronic disease risk factors using factor analysis with oblique rotation and three-factor solution allowing for polychoric correlation between the variables (Additional file 1: Table S1) for men and women separately. Based on the indices, participants were arbitrarily categorised with either a low load of risk factors (the lowest $60 \%$ ) or with a high load of risk factors (the top 40\%). About $29.6 \%$ of 45,616 individuals in this study had missing data in at least one of the variables used in the analysis, mainly from the alcohol dependency variable $(22.8 \%$ missing values), the social participation variable $(4.5 \%)$, the smokeless tobacco use variable (4.2\%) and the diabetes variable (4\%). We therefore conducted multiple imputations with a total of ten imputations - based on Rubin's approach for the imputation of missing data [19].

We presented descriptive statistics as to the socioeconomic and social capital indicators of the study participants. We conducted Cox-proportional hazard regression analysis to analyse the hazard ratio of living alone among adults on their subsequent mortality, controlling for sociodemographic variables, chronic disease risk factor load and social capital variables. We tested for the interaction between living arrangement and social capital variables and found no significant interaction (data not shown).

As a sensitivity analysis, we also conducted a similar analysis for all population age 50 and over who lived in the Västerbotten County in 1990 and followed them until death or censored in October 2015. For this analysis, we did not control for chronic disease risk factors and access to social capital as this information were not available in the register data. All analyses were conducted in Stata 15 (Stata Corp Texas, 2017).

\section{Results}

We analysed a total of 45,616 individuals aged 50 and 60 years who had first participated in the VIP during the years 1990-2006, with a total of 7019 deaths observed within 445,823 person-years of observation. These yielded an overall mortality rate of 19.1 per 1000 person-years in men and 12.7 in women (Table 1). As the mortality rates differed between those with and without missing data (Additional file 1: Table S2), we used the multiplied imputed data for subsequent analyses.

The characteristics of the study respondents are summarised in Table 2. About 19\% of men and 24\% of women had a high education. We observed a high proportion of the individuals in the VIP cohort who lived in partnership with children at home (40\% among men and 30\% among women) and were fully employed (about $72 \%$ in men and women). About $22 \%$ men and $21 \%$ women reported living in a single-family household with no children at home. About $40 \%$ of men and $44 \%$ of women in the VIP study reported having no access to social participation, while in contrast, $71 \%$ of men and women reported having high access to the informal socialising form of social capital.

In the Cox regression analysis (Table 3), we observed that those who lived alone were at a much higher risk of death compared to those who were in a partnership and with children at home (hazard ratio/HR $=1.38,95 \% \mathrm{CI}=1.26-$ 1.50 in men and $\mathrm{HR}=1.27,95 \% \mathrm{CI}=1.13-1.42$ in women), after controlling for other sociodemographic variables, as well as social capital and risk factor burden. Men who were single parents with children at home also exhibited an approximately similar level of risk of mortality $(\mathrm{HR}=1.26$, $95 \% \mathrm{CI}=1.06-1.49$ ). We observed that men without full employment, or those who were pensioners, had a significantly higher risk of mortality when compared to their counterparts who had full employment. Among women, the effects were significant for pensioners and those with intermittent employment. A significant association between no/low access to social participation and mortality was observed in both men $(\mathrm{HR}=1.12,95 \% \mathrm{CI}=1.04-1.20)$ and women $(\mathrm{HR}=1.10,95 \% \mathrm{CI}=1.01-1.19)$, but not observed for a lack of informal socialising after adjustments were made for other factors.

The analysis on the whole Västerbotten population age 50 and over also showed a similar higher risk of mortality among individuals who lived alone with a hazard ratio of $1.32(95 \% \mathrm{CI}=1.26-1.37)$ among men and $1.20(95 \% \mathrm{CI}=$ 1.14-1.26) among women (Additional file 1: Table S3), which were quite similar to the hazard ratio observed among the VIP participants when also controlled chronic disease risk factors and access to social capital.

\section{Discussions}

In this study, we found an association between living alone and mortality among the older population in Västerbotten County. This phenomenon could be an important public health issue in light of the ageing transition and how society and its health care systems adapt to it. Additional analyses using the Linnaeus database indicated an increase in the proportion of adults aged 40+ who lived alone in the Västerbotten County, from 29\% in 1990 to $40 \%$ in 2006. During the same period, the proportion of divorcees and widow/widower adults aged 40+ increased. In 2006, a majority of older people aged 60-69 years in Västerbotten County reported living alone (data not shown). Similar trends were observed in the US $[2,20]$. More US men aged $65+$ preferred to live with their spouse, in contrast 
Table 1 Mortality rate in the VIP study population

\begin{tabular}{|c|c|c|c|}
\hline \multirow[t]{2}{*}{ Variables } & \multicolumn{3}{|c|}{ VIP participants } \\
\hline & $\begin{array}{l}50 \\
(n=25,441)\end{array}$ & $\begin{array}{l}60 \\
(n=20,175)\end{array}$ & $\begin{array}{l}\text { Total } \\
(n=45,616)\end{array}$ \\
\hline \multicolumn{4}{|l|}{ Men } \\
\hline Number population & 12,450 & 9776 & 22,226 \\
\hline Number of deaths (up to Oct 31, 2015) & 1292 & 2765 & 4057 \\
\hline Total follow-up (year) & 119,657 & 92,800 & 212,457 \\
\hline Mortality rate per 1000 person-year & 10.8 & 29.8 & 19.1 \\
\hline \multicolumn{4}{|l|}{ Women } \\
\hline Number population & 12,991 & 10,399 & 23,390 \\
\hline Number of deaths (up to Oct 31, 2015) & 909 & 2053 & 2962 \\
\hline Total follow-up (year) & 129,012 & 104,354 & 233,366 \\
\hline Mortality rate per 1000 person-year & 7.1 & 19.7 & 12.7 \\
\hline
\end{tabular}

with women in a similar age group. Along with the changes in living arrangements among the US population, the network size decreased, and social isolation increased during 1985-2004 [20]. Ruggles reported a substantial decline in the proportion of older people aged 65+ who resided with their children age 18+ between 1850 and 1990 (54\% among whites and 28\% among blacks) and concluded that changes in living arrangements were due to the increased opportunities for the younger generation in the last century [2].

The causal pathways of living alone and mortality are multi-faceted. People who lived alone tended to have a more restricted social network (social isolation), poorer health, and a higher risk of mortality. In the present study, we found that the unemployed and pensioners had a higher mortality risk compared to other population groups. Beyond retirement age, older people's families and social networks tend to shrink. An increasing trend towards the formation of smaller offspring nuclear families, the progression towards widowhood, worsening health conditions, limitations on physical mobility, and natural selection of mortality among peers, exposed older people to the threat of physical and social isolation. Our findings strengthen the evidence that the association between living alone and mortality persisted significantly, even after controlling for social participation and informal socialising. Social participation was consistently significant in its association with mortality for both men and women, but not with regards to informal socializing.

Using the English Longitudinal Study of Ageing, Shankar et al. showed that older people who experienced social isolation and loneliness, especially those with a lower education, had poorer cognitive function within a four-year follow-up period [21]. Steptoe et al. used the same dataset and observed that social isolation was associated with a higher risk of mortality, but not due to the emotional experience of loneliness. Though both social isolation and loneliness were associated with a poorer quality of life, unlike social isolation, the effect of loneliness was not independent of demographic variables and health problems [22]. A meta-analytic review published in 2010 synthesised findings across 148 studies globally and concluded that people with stronger social relationships have a $50 \%$ increased likelihood of survival, and this positive effect is comparable to the negative effect of smoking, obesity, and physical inactivity [7]. Other studies have reported an interaction between a feeling of loneliness and living alone [23, 24], and suggested that the absence of depression and a functional social network are significant predictors of not feeling lonely despite living alone [23]; particularly if social support from friends following widowhood exist [24]. In this study, we observed that the effect of living alone increased among men and women when chronic NCD risk factors and social capital were controlled in the subset analysis.

Our findings provide strong evidence for public and health policy-making that society has to be prepared for the negative outcomes of growing isolation and loneliness when the number of people living alone is increasing. Therefore, special measures to care for and assist lonely older people are necessary through proactive housing policies and the design of neighbourhoods. Theoretical-based interventions, such as those based on self-management of a well-being theory [25] or the formation of social ties [26], especially those ties involving social activity and support within a group format, are more effective in preventing social isolation among older people [27]. 
Table 2 Socio-demographic characteristics and social capital levels in the VIP participants

\begin{tabular}{|c|c|c|}
\hline \multirow[t]{2}{*}{ Variables } & \multicolumn{2}{|c|}{ VIP participants aged 50 and 60} \\
\hline & $\begin{array}{l}\text { Men }(n=22,226) \\
\%(95 \% \mathrm{Cl})\end{array}$ & $\begin{array}{l}\text { Women }(n=23,390) \\
\%(95 \% \text { Cl) }\end{array}$ \\
\hline \multicolumn{3}{|l|}{ Family type and living arrangement (\%) } \\
\hline In partnership with children at home & $39.3(38.7-40.0)$ & $29.9(29.3-30.5)$ \\
\hline In partnership without children at home & $34.8(34.2-35.4)$ & $42.8(42.1-43.4)$ \\
\hline Single parent with children at home & $4.0(3.8-4.3)$ & $6.7(6.4-7.0)$ \\
\hline Single with no children at home & $21.8(21.3-22.4)$ & $20.6(20.1-21.2)$ \\
\hline \multicolumn{3}{|l|}{ Employment status (\%) } \\
\hline Fully employed & $72.1(71.5-72.6)$ & $72.2(71.6-72.8)$ \\
\hline Employed with unemployed & $5.5(5.2-5.8)$ & $5.8(5.5-6.1)$ \\
\hline Mostly unemployed & $3.4(3.2-3.7)$ & $2.0(1.8-2.1)$ \\
\hline Fully unemployed & $4.0(3.8-4.3)$ & $3.4(3.2-3.6)$ \\
\hline Partly pensioner & $5.0(4.7-5.3)$ & $4.9(4.6-5.1)$ \\
\hline Fully pensioner & $9.9(9.5-10.3)$ & $11.7(11.3-12.1)$ \\
\hline \multicolumn{3}{|l|}{ Education level (\%) } \\
\hline Basic education & $33.4(32.8-34.0)$ & $27.2(26.7-27.8)$ \\
\hline Middle education & $47.9(47.3-48.6)$ & $48.6(48.0-49.3)$ \\
\hline High education & $18.7(18.2-19.2)$ & $24.2(23.6-24.7)$ \\
\hline \multicolumn{3}{|l|}{ Social participation (\%) } \\
\hline High access & $23.8(23.2-24.4)$ & $26.0(25.5-26.6)$ \\
\hline Medium access & $23.1(22.6-23.7)$ & $20.3(19.8-20.8)$ \\
\hline Low access & $13.1(12.6-13.5)$ & $9.9(9.5-10.3)$ \\
\hline No access & $40.0(39.4-40.7)$ & $43.8(43.1-44.5)$ \\
\hline \multicolumn{3}{|l|}{ Informal socializing (\%) } \\
\hline High access & $70.6(70.0-71.2)$ & 71.3 (70.7-71.9) \\
\hline Medium access & $17.7(17.2-18.2)$ & $17.1(16.6-17.6)$ \\
\hline Low access & $8.0(7.6-8.3)$ & $7.6(7.3-8.0)$ \\
\hline No access & $3.8(3.5-4.0)$ & $3.9(3.7-4.2)$ \\
\hline
\end{tabular}

In addition to the variables above, we also had information about risk factor quintiles for individuals who participated in the VIP program during 1990-2006. As the risk factor quintiles were approximately equally distributed; hence we do not present the information in this table. The proportions and $95 \%$ confidence intervals for the VIP participants are based on imputed data using the Rubin approach. See text in the Methods section for more details

\section{Strengths and limitations of the study}

Our study has some strengths, in that we were able to follow-up the VIP population for 26 years and assess the impact of its living arrangements on its mortality, while controlling for several socio-economic, biological risk factor and social capital variables. Multiple imputations were conducted to deal with missing data observed in the VIP study and to reduce the bias. We also acknowledge some unavoidable limitations in this study. Swedish register data only includes information about cohabitants who have common children. This means that the number of non-married cohabitants is underestimated and the number living alone is slightly overestimated. But since this study focuses on the older generation where cohabitation without children and without marriage is rare, this has a marginal effect on the conclusion from our study. Second, we could not assess the effect of the feeling of loneliness on mortality within this study as an independent factor, because the VIP does not measure subjective loneliness. Third, this study did not focus on exploring the pathway between living alone and mortality, for example, if individuals who live alone are more prone to adopt certain lifestyle behaviours, or might have more medical conditions, poorer physical and cognitive functions, which in turn increase their mortality risk. Understanding of the mechanisms of living alone and mortality will allow tailoring intervention strategies to prevent the negative impacts of living alone among the older population. 
Table 3 Hazard ratio of mortality related to living alone among the VIP participants

\begin{tabular}{|c|c|c|}
\hline \multirow[t]{2}{*}{ Variables } & \multicolumn{2}{|c|}{ VIP participants aged 50 and 60 recruited during 1990-2006 } \\
\hline & $\begin{array}{l}\text { Men }(n=22,226) \\
\text { Hazard Ratio } \\
\text { (95\% Confidence Interval) }\end{array}$ & $\begin{array}{l}\text { Women }(n=23,390) \\
\text { Hazard Ratio } \\
(95 \% \text { Confidence Interval) }\end{array}$ \\
\hline \multicolumn{3}{|l|}{ Age group } \\
\hline 50 years & Reference & Reference \\
\hline 60 years & $1.84(1.70-1.98)$ & $1.66(1.52-1.82)$ \\
\hline \multicolumn{3}{|l|}{ Family type and living arrangement } \\
\hline In partnership with children at home & Reference & Reference \\
\hline In partnership without children at home & $1.08(0.99-1.17)$ & $1.04(0.94-1.15)$ \\
\hline Single parent with children at home & $1.26(1.06-1.49)$ & $0.95(0.79-1.14)$ \\
\hline Single with no children at home & $1.38(1.26-1.50)$ & $1.27(1.13-1.42)$ \\
\hline \multicolumn{3}{|l|}{ Employment status } \\
\hline Fully employed & Reference & Reference \\
\hline Intermittent employment & $1.29(1.12-1.49)$ & $1.06(0.88-1.26)$ \\
\hline Mostly unemployed & $1.36(1.16-1.61)$ & $1.61(1.22-2.12)$ \\
\hline Fully unemployed & $1.35(1.15-1.58)$ & $1.12(0.93-1.35)$ \\
\hline Partly pensioner & $1.14(1.01-1.28)$ & $1.48(1.28-1.70)$ \\
\hline Fully pensioner & $1.58(1.45-1.72)$ & $1.53(1.39-1.68)$ \\
\hline \multicolumn{3}{|l|}{ Education level } \\
\hline High education & Reference & Reference \\
\hline Middle education & $1.11(1.00-1.23)$ & $1.11(0.98-1.24)$ \\
\hline Basic education & $1.05(0.94-1.17)$ & $1.06(0.93-1.19)$ \\
\hline \multicolumn{3}{|l|}{ 1st risk factor load } \\
\hline Low risk load (60\% lowest) & Reference & Reference \\
\hline High risk load (40\% highest) & $1.02(0.93-1.11)$ & $1.01(0.89-1.15)$ \\
\hline \multicolumn{3}{|l|}{ 2nd risk factor load } \\
\hline Low risk load (60\% lowest) & Reference & Reference \\
\hline High risk load (40\% highest) & $1.11(1.03-1.21)$ & $1.09(0.93-1.27)$ \\
\hline \multicolumn{3}{|l|}{ 3rd risk factor load } \\
\hline Low risk load (60\% lowest) & Reference & Reference \\
\hline High risk load (40\% highest) & $1.08(1.00-1.17)$ & $1.20(1.08-1.32)$ \\
\hline \multicolumn{3}{|l|}{ Social participation } \\
\hline High / medium access & Reference & Reference \\
\hline Low / No access & $1.12(1.04-1.20)$ & $1.10(1.01-1.19)$ \\
\hline \multicolumn{3}{|l|}{ Informal socializing } \\
\hline High / medium access & Reference & Reference \\
\hline Low / No access & $1.05(0.95-1.17)$ & $1.05(0.94-1.17)$ \\
\hline
\end{tabular}

Only 95\% Cls which have significance are bolded.

\section{Conclusions}

Association between living alone and mortality among the adult population aged 50 and 60 years old in Västerbotten County in Sweden was observed and the association persisted even after controlling for chronic disease risk factors and social capital. Therefore, it is important for policy makers to reduce the health and societal impacts of living alone, by focusing on designing supportive housing structures and neighbourhoods that will provide good social support for people who live alone, especially older people, in order to prevent the negative health impacts of living alone. 


\section{Supplementary information}

Supplementary information accompanies this paper at https://doi.org/10. 1186/s12877-019-1330-9

Additional file 1: Table S1. Factor loadings of components of chronic disease risk factors among men and women in the VIP. Table S2. Mortality rate among VIP participants with complete and incomplete data in the analysis. Table S3. Hazard ratio of mortality related to living alone among the Västerbotten population in 1990.

\section{Abbreviations}

BMI: Body mass index; HR: Hazard ratio; VIP: Västerbotten Intervention Program

\section{Acknowledgements}

Not applicable.

\section{Authors' contributions}

NN prepared the initial draft of the manuscript. NN and AS conducted the statistical analysis. LW and GM provided critical inputs to the manuscript. All authors contributed to the interpretation of the statistical results, and all authors approved the final draft of the paper for submission.

\section{Funding}

The research was funded by the Swedish Foundation for Humanities and Social Sciences (Riksbankens jubileumsfond), the project Family network, life style and health. Grant number: P11-1058:1. NN and AS were also supported by the project "Paths to Healthy and Active Ageing" funded by the Swedish Research Council for Health, Working Life and Welfare, Dnr: 2013-2056. The Network for International Longitudinal Studies on Ageing, funded by the Swedish Forte Network Grant (Dnr: 2015-01499) supports the publication of this paper. The funding bodies did not have any role in the design of the study and collection, analysis, and interpretation of data and in writing the manuscript.

\section{Availability of data and materials}

The Linnaeus database contains anonymised individual data from surveys and Swedish population register data, which is maintained in secure server located at Umeå University and are not publicly available.

\section{Ethics approval and consent to participate}

We obtained the ethical approval for the VIP examination (Dnr 08-131 M) and the Linnaeus Database (Dnr 07. 142Ö) from the regional ethics committee in Umeå. All the VIP participants provide written consent prior to their participation in the survey.

\section{Consent for publication}

This manuscript does not contain any individual person's data that needing a consent for publication.

\section{Competing interests}

The authors declare that they have no competing interests.

\section{Author details}

'Department of Epidemiology and Global Health, Faculty of Medicine, Umeå University, Umeå, Sweden. ${ }^{2}$ Centre for Demographic and Ageing Research, Umeå University, Umeå, Sweden. ${ }^{3}$ Global Health, School of Public Health and Community Medicine, Institute of Medicine, Sahlgrenska Academy, University of Gothenburg, Gothenburg, Sweden. "Department of Geography, Umeå University, Umeå, Sweden.

Received: 28 January 2019 Accepted: 29 October 2019

Published online: 06 January 2020

\section{References}

1. United Nation Department of Economic and Social Affairs. Living arrangement of older persons around the world. New York: UNDESA / Population Division; 2005.

2. Ruggles S. The decline of intergenerational coresidence in the United States, 1850 to 2000. Am Sociol Rev. 2007;72:964-89.
3. Tomassini C, Glaser K, Wolf DA, Broese van Groenou MI, Grundy E. Living arrangements among older people: an overview of trends in Europe and the USA. Popul Trends. 2004;115:24-34

4. Eurostat. Over half of Sweden's households made up of one person. Eurostat; 2018. Available from: https://ec.europa.eu/eurostat/web/productseurostat-news/-/DDN-20170905-1?inheritRedirect=true. Cited 9 Jan 2019

5. Staehelin K, Schindler C, Spoerri A, Zemp Stutz E. Marital status, living arrangement and mortality: does the association vary by gender? J Epidemiol Community Health. 2012;66:e22.

6. Koskinen S, Joutsenniemi K, Martelin T, Martikainen P. Mortality differences according to living arrangements. Int J Epidemiol. 2007;36:1255-64.

7. Holt-Lunstad J, Smith TB, Layton JB. Social relationships and mortality risk: a meta-analytic review. PLoS Med. 2010;7:e1000316.

8. Xiu-Ying H, Qian C, Xiao-Dong P, Xue-Mei Z, Chang-Quan H. Living arrangements and risk for late life depression: a meta-analysis of published literature. Int J Psychiatry Med. 2012;43:19-34.

9. Shankar A, McMunn A, Banks J, Steptoe A. Loneliness, social isolation, and behavioral and biological health indicators in older adults. Health Psychol. 2011:30:377-85.

10. Pantell M, Rehkopf D, Jutte D, Syme SL, Balmes J, Adler N. Social isolation: a predictor of mortality comparable to traditional clinical risk factors. Am J Public Health. 2013;103:2056-62.

11. Stickley A, Koyanagi A, Roberts B, et al. Loneliness: its correlates and association with health behaviours and outcomes in nine countries of the former Soviet Union. PLoS One. 2013;8:e67978.

12. Nicholson NR. A review of social isolation: an important but underassessed condition in older adults. J Prim Prev. 2012;33:137-52.

13. Vlachantoni A, Shaw RJ, Evandrou M, Falkingham J. The determinants of receiving social care in later life in England. Ageing Soc. 2015;35:321-45.

14. Malmberg G, Nilsson LG, Weinehall L. Longitudinal data for interdisciplinary ageing research. Design of the Linnaeus Database. Scand J Public Health. 2010;38:761-7.

15. Norberg M, Wall S, Boman K, Weinehall L. The Västerbotten intervention Programme: background, design and implications. Glob Health Action. 2010;3:4643.

16. Ewing JA. Detecting alcoholism. The CAGE questionnaire. JAMA. 1984;252:1905-7.

17. Ng N, Soderman K, Norberg M, Ohman A. Increasing physical activity, but persisting social gaps among middle-aged people: trends in northern Sweden from 1990 to 2007. Glob Health Action. 2011:4:6347.

18. Eriksson M, Ng N. Changes in access to structural social capital and its influence on self-rated health over time for middle-aged men and women: a longitudinal study from northern Sweden. Soc Sci Med. 2015;130:250-8.

19. Rubin DB. Multiple imputation for nonresponse in surveys. New Jersey: Wiley; 1987.

20. McPherson M, Smith-Lovin L, Brashears ME. Social isolation in America: changes in core discussion networks over two decades. Am Sociol Rev. 2006;71:353-75.

21. Shankar A, Hamer M, McMunn A, Steptoe A. Social isolation and loneliness: relationships with cognitive function during 4 years of follow-up in the English longitudinal study of ageing. Psychosom Med. 2013;75:161-70.

22. Steptoe A, Shankar A, Demakakos P, Wardle J. Social isolation, loneliness, and all-cause mortality in older men and women. Proc Natl Acad Sci USA 2013;110:5797-801.

23. Zebhauser A, Baumert J, Emeny RT, Ronel J, Peters A, Ladwig KH. What prevents old people living alone from feeling lonely? Findings from the KORA-age-study. Aging Ment Health. 2015;19:773-80.

24. Utz RL, Swenson KL, Caserta M, Lund D, deVries B. Feeling lonely versus being alone: loneliness and social support among recently bereaved persons. J Gerontol B Psychol Sci Soc Sci. 2014:69:85-94.

25. Steverink N, Lindenberg S, Slaets JPJ. How to understand and improve older people's self-management of wellbeing. Eur J Ageing. 2005:2:235-44.

26. Cohen S, Gottlieb BH, Underwood LG. Social relationships and health. In: Cohen S, Underwood LG, Gottlieb BH, editors. Social support measurement and intervention: a guide for health and social psychologists. New York: Oxford University Press; 2000

27. Dickens AP, Richards SH, Greaves CJ, Campbell JL. Interventions targeting social isolation in older people: a systematic review. BMC Public Health. 2011;11:647.

\section{Publisher's Note}

Springer Nature remains neutral with regard to jurisdictional claims in published maps and institutional affiliations. 